Patientenberatung

\section{Entscheidungshilfe Prostatakrebs}

Zuwarten, Totaloperation oder Bestrahlung? Angesichts des breiten Behand-

lungsspektrums beim nicht metastasierten Prostatakarzinom fällt die Therapieentscheidung häufig besonders schwer. Nun hat die PatientenAkademie der Deutschen Urologen eine onlinebasierte interaktiv personalisierte Entscheidungshilfe für Patienten mit nicht metastasiertem Prostatakarzinom entwickelt, die Betroffene und ihre betreuenden Urologen bei der Wahl der individuell richtigen

Therapie unterstützt.

„Der ,Clou‘ unseres Angebotes liegt in der interaktiven Personalisierung nach onkologischem Risiko, Erektionsfunktion und Begleiterkrankungen. Das kann keine noch so gute Broschüre leisten! “, sagt Projektleiter PD Dr. Dr. Johannes Huber aus Dresden.

Im deutschsprachigen Raum ist das Konzept bislang einzigartig: Dabei bietet der Urologe seinem Prostatakrebspatienten kurz nach der Erstdiagnose die Nutzung der personalisierten Entscheidungshilfe an und stellt ihm einen individuellen $\mathrm{Zu}$ gangscode sowie 8 klinische Angaben zur Verfügung. Auf Basis seiner Eingaben erhält der Patient leitliniengerechte personalisierte Informationen zu seiner Erkrankung und den Behandlungsmöglichkeiten. So kann sich der Betroffene in Ruhe zuhause mit Personen seines Vertrauens informieren. Alle Inhalte sind als patientenorientierte Erklärvideos aufbereitet. In das anschließende Arztgespräch bringt der Patient dann eine standardisierte $\mathrm{Zu}$ sammenfassung seiner Daten mit. Dazu zählen Angaben zu Komorbidität, Erektions- und Miktionsfunktion, psychischer Belastung sowie zu subjektiven Prioritäten, von denen die individuelle Beratung zusätzlich profitiert. Für Urologen stellen diese gezielte Vorbereitung und ihre automatische Dokumentation eine Entlastung im klinischen Alltag dar. Weitere Informationen sind verfügbar unter www. entscheidungshilfe-prostatakrebs.info.

Als gemeinsame Initiative der DGU und des BDU hat die PatientenAkademie einen breiten Rückhalt in der deutschen Urologie. Das Projektteam war überdies interdisziplinär besetzt. Die Vorsitzende der PatientenAkademie Prof. Sabine Kliesch appelliert: „Ich möchte alle Kollegen und Kolleginnen ausdrücklich ermutigen, die ,Entscheidungshilfe Prostatakrebs' zu testen und sie ihren Patienten anzubieten. Dieses Leuchtturmprojekt ist ein Angebot aus urologischer Hand und transportiert ohne alltäglichen Mehraufwand die Inhalte der S3-Leitlinie Prostatakarzinom zu unseren Patienten." Die technische Umsetzung erfolgte durch die Firma ASD Concepts mit freundlicher Unterstützung von Takeda Pharma.

„Etwa 2 von 3 Betroffenen werden unser Angebot nutzen können“, schätzt Huber. „Denn mittlerweile liegt die Internetnutzung der 70-Jährigen in dieser Größenordnung. Einige weitere werden sich sicher von ihren Kindern oder Enkeln helfen lassen." Die Effekte der Entscheidungshilfe Prostatakrebs sollen im Verlauf wissenschaftlich evaluiert werden. Hieraus kann zusätzlich eine sehr wertvolle Versorgungsforschungsstudie entstehen. In erster Linie sollen jedoch Patienten und Urologen von dem Angebot profitieren.

Interessierte können das innovative Beratungsportal für Patienten mit nicht metastasiertem Prostatakarzinom unter www. entscheidungshilfe-prostatakrebs.de mithilfe eines Testzugangs kennenlernen.

Nach einer Pressemitteilung (DGU/BDU) 